\title{
Erratum to: Remarks on lines and minimal rational curves
}

\author{
MOK Ngaiming ${ }^{1} \&$ SUN XiaoTao ${ }^{2, *}$ \\ ${ }^{1}$ Department of Mathematics, The University of Hong Kong, Pokfulam Road, Hong Kong, China; \\ ${ }^{2}$ Academy of Mathematics and Systems Science, Chinese Academy of Sciences, Beijing 100190, China \\ Email:nmok@hkucc.hku.hk,xsun@math.ac.cn
}

Erratum to: Science in China Series A: Mathematics, April 2009 Vol. 52 No.4: 617-630 doi: $10.1007 / \mathrm{s} 11425-009-0038-2$

There is a mistake in the proof of [1, Lemma 2.2], which occurs in 4-th line at [1, p. 619], where we state: The last inequality holds because $r_{2}^{\prime}-\frac{r}{(r, d)} \operatorname{deg}\left(V_{2}^{\prime *} \otimes V_{2}\right)<r_{2}$ and is divisible by $r_{2}$, thus it must be negative. Namely, in [1], we claimed that

$$
r_{2}^{\prime}-\frac{r}{(r, d)} \operatorname{deg}\left(V_{2}^{\prime *} \otimes V_{2}\right)<0
$$

(which implies the stability of $V$ ). However, it could be zero, i.e.,

$$
r_{2}^{\prime}-\frac{r}{(r, d)} \operatorname{deg}\left(V_{2}^{\prime *} \otimes V_{2}\right) \leqslant 0
$$

which only implies the semistability of $V$. Thus the corrected version of Lemma 2.2 in [1] should be the following lemma.

Lemma 2.2 Let $0 \rightarrow V_{1} \rightarrow V \rightarrow V_{2} \rightarrow 0$ be a nontrivial extension of vector bundles on $C$. Let $r_{i}=\operatorname{rk}\left(V_{i}\right), d_{i}=\operatorname{deg}\left(V_{i}\right)(i=1,2), r=\operatorname{rk}(V), d=\operatorname{deg}(V)$ be the rank and degree, respectively. Then, when $r_{1} d-d_{1} r=(r, d)$, we have

(1) stability of $V$ implies the stability of $V_{1}$ and $V_{2}$;

(2) stability of $V_{1}$ and $V_{2}$ implies that $V$ is semi-stable and it is stable if $r_{2}<\frac{r}{(r, d)}$.

With this correction, morphism $(2.2)$ at $[1$, p. 620] should be

$$
\Phi: P\left(r_{1}, d_{1}\right) \rightarrow \mathcal{S U}_{C}(r, \mathcal{L}) \supseteq \mathcal{S U}_{C}(r, \mathcal{L})^{s}=M .
$$

Replacing $P\left(r_{1}, d_{1}\right)$ by $\Phi^{-1}(M)$, all of our results in [1] hold unchanged.

\section{References}

1 Mok N, Sun X T. Remarks on lines and minimal rational curves. Sci China Ser A, 2009, 52: 617-630

The online version of the original article can be found at http://dx.doi.org/10.1007/s11425-009-0038-2

${ }^{*}$ Corresponding author

(C) Science China Press and Springer-Verlag Berlin Heidelberg 2014

math.scichina.com link.springer.com 